\title{
Fenestrated A1 in Ruptured Anterior Communicating Artery Aneurysm: A Case Report
}

\author{
Pankaj Raj Nepal $^{1}$, Dinesh Kumar Thapa ${ }^{1}$, Robin Bhattrai ${ }^{1}$, Jagat Narayan Rajbanshi ${ }^{1}$
}

${ }^{1}$ Department of Neurosurgery, B and C Medical College Teaching Hospital and Research Center, Birtamode, Jhapa, Nepal

\section{Correspondence:}

Dr. Pankaj Raj Nepal, FCPS

Deputy Medical Director and Head of Department,

Department of Neurosurgery,

$\mathrm{B}$ and C Medical College Teaching Hospital and Research Center,

Birtamode, Jhapa, Nepal

Email: pankajrajnepal@gmail.com

\begin{abstract}
There is a lot of variation around the anterior communicating artery (Acom) and various classification systems have been quoted many times in literatures. Here we have reported a rare case of a 52year-old woman who had ruptured Acomartery aneurysm. The angiography also showed a fenestration of the A1 segment of anterior cerebral artery.
\end{abstract}

$\Lambda$

nomalous variation of the anterior communicating artery (Acom) has been described many times in the literature and various classifications have been quoted. ${ }^{1}$ As there is a lot of variation around the Acom artery and because of its location and projection of the aneurysm, Acom artery aneurysm is most difficult to operate among other anterior circulating aneurysms. ${ }^{2}$ Here we have reported the case of a rare fenestration of the Al segment of anterior cerebral artery associated with ruptured Acom artery aneurysm.

\section{Methods and Materials:}

\section{Case description:}

A 52- year-old right-handed woman presented to our emergency department with a history of sudden onset of severe headache for 5 hours, and it was associated with multiple episodes of vomiting. There was no history of loss of consciousness, trauma, and fever. On arrival her blood pressure was $180 / 110 \mathrm{~mm}$ of $\mathrm{Hg}$, she was opening eyes to speech, verbally confused, and obeying command.
Her pupils were bilateral $3 \mathrm{~mm}$ reacting to light and was moving all the four limbs. CT scan of her head showed diffuse subarachnoid hemorrhage more localized in perioptic cistern and anterior interhemispheric region. Her CT angiography showed Acom artery aneurysm pointing anteriorly and there was a fenestration of the right A1 segment of the anterior cerebral artery.

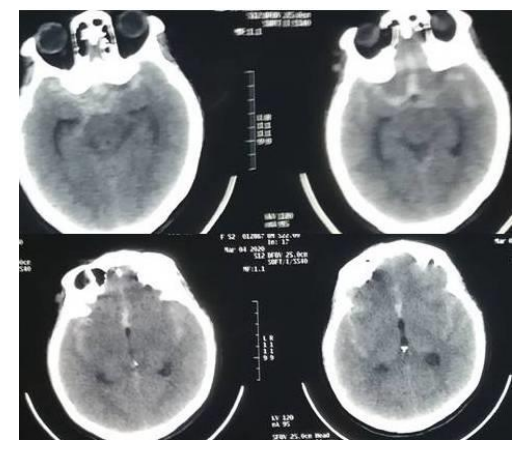

Figure 1: CT scan showed diffuse subarachnoid hemorrhage 


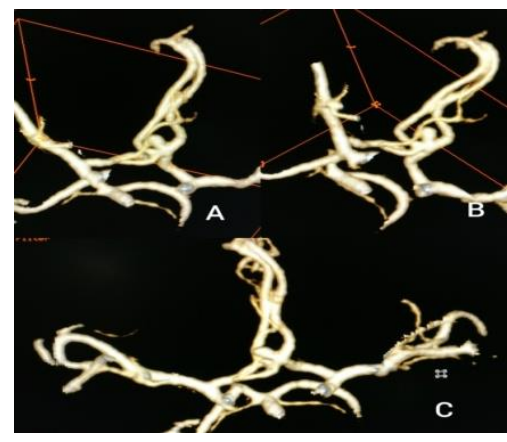

Figure 2: CT angiography showed anterior pointing Acom aneurysm with fenestrated right A1 segment.

She underwentright pterional craniotomy and clipping of aneurysm. The osteoplastic bone flap was raised with three burr holes, and the lesser wing of the sphenoid bone was flushed down to the meningo-orbital band, which was detached. Dura was opened curvilinearly and was based inferiorly. Optico-carotid cistern was opened to release CSF from the basal cistern.

Microscopic dissection was carried out to identify bilateral A1, including the right fenestrated part, it required the resection of a part of the gyrus-rectus and finally, bilateral A2 were also identified. During the dissection around the neck of the aneurysm, it got ruptured so a tentative clipping was done after placing the temporary clips in bilateral A1. And finally, the permanent clips were placed just at the neck preserving all the proximal and distal parent vessels. The patency of the vessels was confirmed by visual inspection.

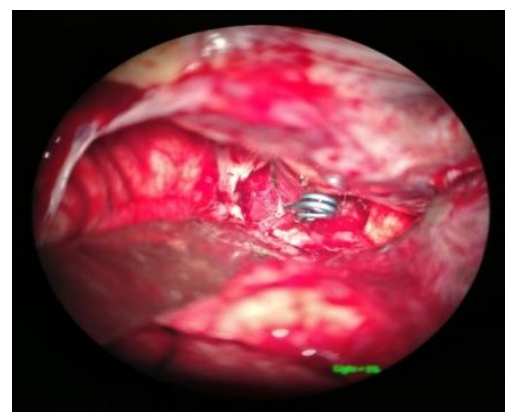

Figure 3: Simple straight aneurysm clip at the neck of the aneurysm.

Postoperatively she was extubated after 7 hours and then she gradually recovered to her previous health. There was no new added focal neurological deficit postoperatively.

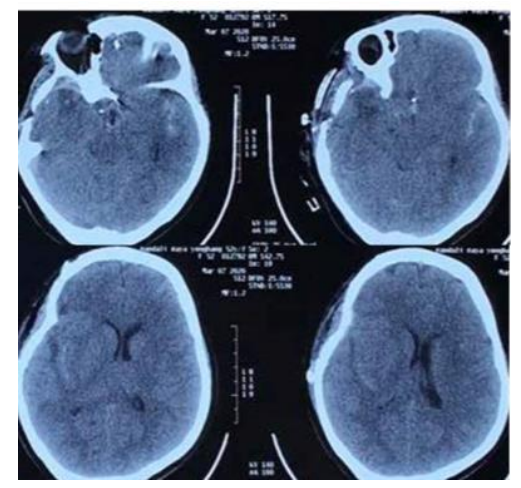

Figure 4: Postoperative CT scan of head.

\section{Discussion:}

Acomartery aneurysms are one of the most difficult aneurysms to clip among other anterior circulating aneurysms. ${ }^{2}$ The difficulty is because of its location behind the gyrus rectus of the frontal lobe, various projection of the aneurysm, and occasionally due to various vascular anomalies that coexist with the aneurysm., ${ }^{2,3}$ Various types of vascular anomalies around the Acom artery that have been described in the literature are typical, atypical, azygous, accessory A2, and fenestrated Acom. ${ }^{1,4,5}$ Along with these variations, A1 fenestrations were also described occasionally in different literatures. ${ }^{6,7,8}$

Similarly, in this case, we have found an fenestrated A1 segment of anterior cerebral artery associated with anterior pointing Acom aneurysm. ${ }^{9,10}$ Clipping of Acom artery aneurysm required proper identification of bilateral A1, A2, and Acom artery so that the clip occluding the neck of the aneurysm will not incorporate those important vessels. ${ }^{2,3}$ In the presence of accessory $\mathrm{A} 1$, it possesses the risk of missing one of the A1 and hence has a risk of misplacement of aneurysm clip. Beside of challenge in clipping, there is also added risk of aneurysm rupture intraoperatively during dissection around the aneurysm. In our case also the aneurysm got ruptured during the last stage of dissection after the identification of proximal and distal vessels. These were dealt with tentative clipping followed by proper neck isolation and final permanent clipping. The patency of surrounding vessels was confirmed by microscopic visual inspection. egneuro, Volume 02, Issue 03, 2020 
Nepal PR et al.

The Projection of aneurysm is also an important factor during the placement of the clip. ${ }^{11}$ Inferior and anterior pointing Acom aneurysm can be usually clipped with straight aneurysm clips, whereas superior pointing aneurysms might require tandem clipping. The posterior pointing Acom aneurysms are difficult ones requiring angle fenestrated clips, where the parent vessel is passed through the fenestration and anterior thalamic perforators need to be preserved during occlusion of the aneurysm neck. In our case, it was anterior pointing Acom aneurysm, so it was possible to clip the neck of aneurysm with a single simple straight aneurysm clip preserving the proximal and distal flow in parent vessels.

\section{Conclusion:}

The presence of accessory A1 can be a rare type of anomalous presentation that may be associated with a ruptured Acom aneurysm.

\section{References:}

1. Uchino A, Nomiyama K, Takase Y, Kudo S. Anterior cerebral artery variations detected by MR angiography. Neuroradiology. 2006 Sep 1;48(9):647-52.

2. Sekhar LN, Natarajan SK, Britz GW, Ghodke B. Microsurgical management of anterior communicating artery aneurysms. Operative Neurosurgery. 2007 Nov 1;61(suppl 5): ONS273-92.

3. Yaşargil MG, Fox JL, Ray MW. The operative approach to aneurysms of the anterior communicating artery. InAdvances and Technical Standards in NeurosurgeryVolume 21975 (pp. 113-170). Springer, Vienna.

4. Karazincir S, Ada E, Sarsilmaz A, Yalçin O, Vidinli B, Sahin E. Frequency of vascular variations and anomalies accompanying intracranial aneurysms. Tanisalvegirisimselradyoloji:

TibbiGoruntulemeveGirisimselRadyolojiDernegiyayinorga ni. 2004 Jun 1;10(2):103-9.

5. Ding X, Nisson PL, James WS, Lawton MT, Ren S, Jia L, Ji H. Aneurysms of the proximal segment of the anterior cerebral artery: a new classification system with corresponding therapeutic options. World Neurosurgery. 2017 Aug 1; 104:291-302.

6. Friedlander RM, Ogilvy CS. Aneurysmal subarachnoid hemorrhage in a patient with bilateral A1 fenestrations associated with an azygos anterior cerebral artery: Case report and literature review. Journal of neurosurgery. 1996 Apr 1;84(4):681-4.

7. Yasargil MG. 4. Operative anatomy. Microneurosurgery. 1984:165-231.

8. Perlmutter D, Rhoton AL. Microsurgical anatomy of the anterior cerebral-anterior communicating-recurrent artery complex. Journal of neurosurgery. 1976 Sep 1;45(3):25972 .

9. Kachhar R, Nair S, Gupta AK. Fenestration of the Proximal Anterior Cerebral Artery (A1) with Aneurysm Manifesting as Subarachnoid Hemorrhage-Case Report-. Neurologia medico-chirurgica. 1998;38(7):40912.

10. Kobayashi S, Yuge T, Sugita Y, Kuratomi A, Katayama M, Iryo O, Kokarashi K, Kuboyama M, Kuramoto S. Azygos anterior cerebral artery aneurysm associated with fenestration of the anterior cerebral artery. The Kurume medical journal. 1987 Feb 20;33(3):149-53.

11. Yang I, Lawton MT. Clipping of complex aneurysms with fenestration tubes: application and assessment of three types of clip techniques. Operative Neurosurgery. 2008 May 1;62(suppl_5): ONS371-9. 